\title{
Monorquidismo em um equino marchador
}

\author{
Monorchidism in a four-beat gaited horse
}

\author{
Jéssica Fontana de Magalhães ${ }^{\mathrm{I}}$ Guilherme Mattos Jardim Costa ${ }^{\mathrm{II}}$ Celina Alves de Oliveira ${ }^{\mathrm{I}}$ \\ Mayara Gomes Corrêa ${ }^{I}$ Sérgio da Silva Rocha Júnior ${ }^{I}$ Suzane Lilian Beier ${ }^{I}$ \\ Heloisa Maria Falcão Mendes ${ }^{I}$ Geraldo Eleno Silveira Alves ${ }^{I}$ Rafael Resende Faleiros $^{I^{*}}$
}

\section{- NOTA -}

\section{RESUMO}

O monorquidismo é um quadro raramente descrito em equinos, que não apresenta descrições prévias na literatura nacional. Trata-se de um equino adulto brasileiro, com oito anos de idade, sem raça definida, porém com características de raças de origem nacional. Por abordagem inguinal do lado esquerdo, localizou-se a túnica vaginal intacta, que, aberta, continha a cauda de um epididimo mal formado, o ducto deferente e um delgado pedículo vascular, mas sem indicios de testículo. Exames histológicos e hormonais confirmaram a ausência de tecido testicular. Conclui-se que os exames histológicos e hormonais são essenciais para o diagnóstico de monorquidismo que, apesar de raro, também pode acometer equinos nacionais.

Palavras-chave: equino, monorquidismo, orquiectomia, diagnóstico, criptorquidismo.

\section{ABSTRACT}

As monorchidism is rarely reported in horses and no case report has been found in the national literature, the objective was to describe a case in an eight-year old Brazilian adult horse. Using an inguinal approach in the left side, an intact tunica vaginalis was found containing the tail of a malformed epididymis, the ductus deferens and a thin vascular pedicle but without evidence of testicle. Histological and hormonal exams confirmed the absence of testicular tissue. In conclusion, histological and hormonal exams are essential for the diagnosis of monorchidism, which although rare, can also affect national horses.

Key words: equine, monorchidism, orchiectomy, diagnosis, cryptorchidism.

Monorquidismo é definido como ausência de um dos testículos, devido à agenesia testicular ou a um acidente vascular testicular unilateral. Raros relatos podem ser encontrados apenas na literatura internacional (PARKS, et al., 1989; SCHUMACHER, 1992; PARKER et al., 1997; STRONG et al., 1997). A retenção ou falha na descida do testículo para a bolsa escrotal é denominada criptorquidismo (PARKER et al., 1997). O criptorquidismo é mais comum do que o monorquidismo, sendo o diagnóstico definitivo obtido apenas após exploração cirúrgica abdominal, remoção do testículo contralateral e dosagem hormonal (ADAMS, 1990; SEARLE et al., 1999). O exame hormonal inclui dosagem de testosterona basal e dosagem de testosterona após estimulação com gonadotrofna coriônica humana (hCG) (ARIGHI et al., 1989; STRONG et al., 1997). A dosagem de testosterona basal seguida da estimulação com hCG em equino com mais de 18 meses de idade é o melhor indicador da presença de tecido testicular vital (SEARLE et al., 1999). Por ser um quadro raramente relatado em equinos, ainda não descrito na literatura nacional, o objetivo foi relatar um caso de monorquidismo em um equino adulto brasileiro, com oito anos de idade, sem raça definida, demonstrando a importância dos exames histológicos e hormonais para o diagnóstico definitivo.

Um garanhão adulto, de oito anos de idade, sem raça definida, foi encaminhado à Clínica Cirúrgica de Grandes Animais do Hospital Veterinário da Universidade Federal de Minas Gerais (HV-UFMG) com suspeita de criptorquidismo

'Clínica Cirúrgica de Grandes Animais, Universidade Federal de Minas Gerais (UFMG), Avenida Antonio Carlos, CP 6627, 31270-901, Belo Horizonte, MG, Brasil. E-mail: faleirosufmg@gmail.com. *Autor para correspondência.

IIDepartamento de Morfologia, UFMG, Belo Horizonte, MG, Brasil. 
unilateral. O paciente não tinha histórico de cirurgia prévia. Durante o exame físico, na palpação externa do escroto e da região inguinal, o testículo direito apresentava tamanho e posição normais, e o testículo esquerdo não foi detectado, mesmo por palpação transretal. O paciente foi encaminhado para cirurgia, sob anestesia geral inalatória e decúbito dorsal, realizou-se acesso inguinal esquerdo através de incisão de pele parainguinal de aproximadamente $12 \mathrm{~cm}$. Por meio de exploração cirúrgica na região do anel inguinal externo, foi encontrada a túnica vaginal intacta, que, aberta, continha a cauda de um epidídimo mal formado, o ducto deferente e um delgado pedículo vascular. O ligamento da cauda do epidídimo estava preservado, unindo-o à túnica vaginal. A incisão da túnica vaginal foi ampliada até o anel vaginal. O ducto deferente foi seguido manualmente até próximo à bexiga e o pedículo vascular foi seguido até a parede dorsal da cavidade peritoneal repetidamente, sem que nenhuma estrutura testicular fosse encontrada. Assim, a cauda do epidídimo e o tecido adjacente foram excisados e realizou-se a síntese do anel inguinal externo, com padrão de sutura em X, preservandose a patência da veia pudenda externa. Logo após, a pele foi suturada com pontos simples separados e realizou-se orquiectomia aberta no lado direito, de forma rotineira.

Com o objetivo de caracterização histológica, o tecido excisado do lado esquerdo e o testículo/epidídimo direitos foram fixados em formol tamponado $10 \%$ e seccionados longitudinalmente com uma lâmina em pequenos fragmentos de 2 a $3 \mathrm{~mm}$ de espessura. Este material foi processado, incluído em paraplast (Sigma
Aldrich), e cortes sequenciais de 5 micrometros foram corados com eosina e hematoxilina $\mathrm{e}$ examinados em microscópio de luz.

Dez dias após a cirurgia, com intuito de verificar a completa ausência de tecido testicular, coletou-se sangue da veia jugular, em tubo sem anticoagulante. Logo em seguida, foi administrado 10.000UI de hCG, IV, e nova amostra de sangue foi obtida da mesma forma uma hora após. As amostras sanguíneas foram encaminhadas ao laboratório Hermes Pardini para dosagem de testosterona, por método de quimioluminescência.

$\mathrm{Na}$ análise histológica, o testículo direito (Figura 1) apresentava histologia normal e completa espermatogênese dentro dos túbulos seminíferos. Do mesmo modo, a histologia do ducto epididimal estava preservada e um alto número de espermatozoides foi observado dentro do lúmem, demonstrando produção espermática normal. Ao contrário, a análise histológica do tecido retido (Figura 2) mostrou a presença do cordão espermático, ducto epididimal e ducto deferente com ausência de estruturas espermáticas nos ductos, entretanto nenhum tecido testicular foi observado.

$\mathrm{Na}$ análise hormonal, as amostras antes e após estimulação por hCG apresentaram índices séricos de testosterona inferiores a $20 \mathrm{ng} \mathrm{dL}^{-1}$, confirmando a ausência de tecido testicular. Além disso, seis meses após a cirurgia, o proprietário relatou que o equino não apresentava qualquer comportamento de garanhão.

São raros os relatos de monorquidismo em equinos na literatura internacional, além de serem relatos antigos (PARKS, et al., 1989;

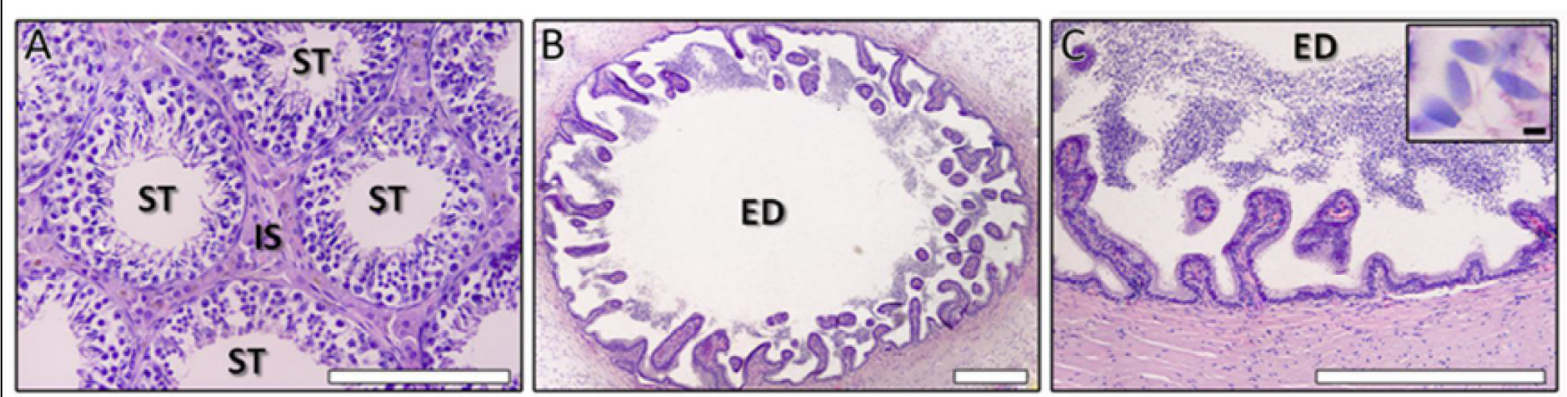

Figura 1 - Fotomicroscopia do testículo e epidídimo eutópicos. Como pode ser observado no painel A, completa espermatogênese é encontrada no epitélio dos túbulos seminíferos, indicando uma produção espermática normal. Os painéis $\mathrm{B}$ e C mostram que a histologia do ducto epididimal está preservada e um elevado número de espermatozoides é observado dentro do lúmen. Na maior ampliação (inserida no painel C), está em evidência a cabeça do espermatozoide com morfologia normal. ST = túbulos seminíferos ; IS = espaço intersticial $; \mathrm{ED}=$ ducto epididimal; $\mathrm{S}=$ espermatozoide. Barra branca $=250 \mu \mathrm{m} ;$ barra preta $=3 \mu \mathrm{m}$. 


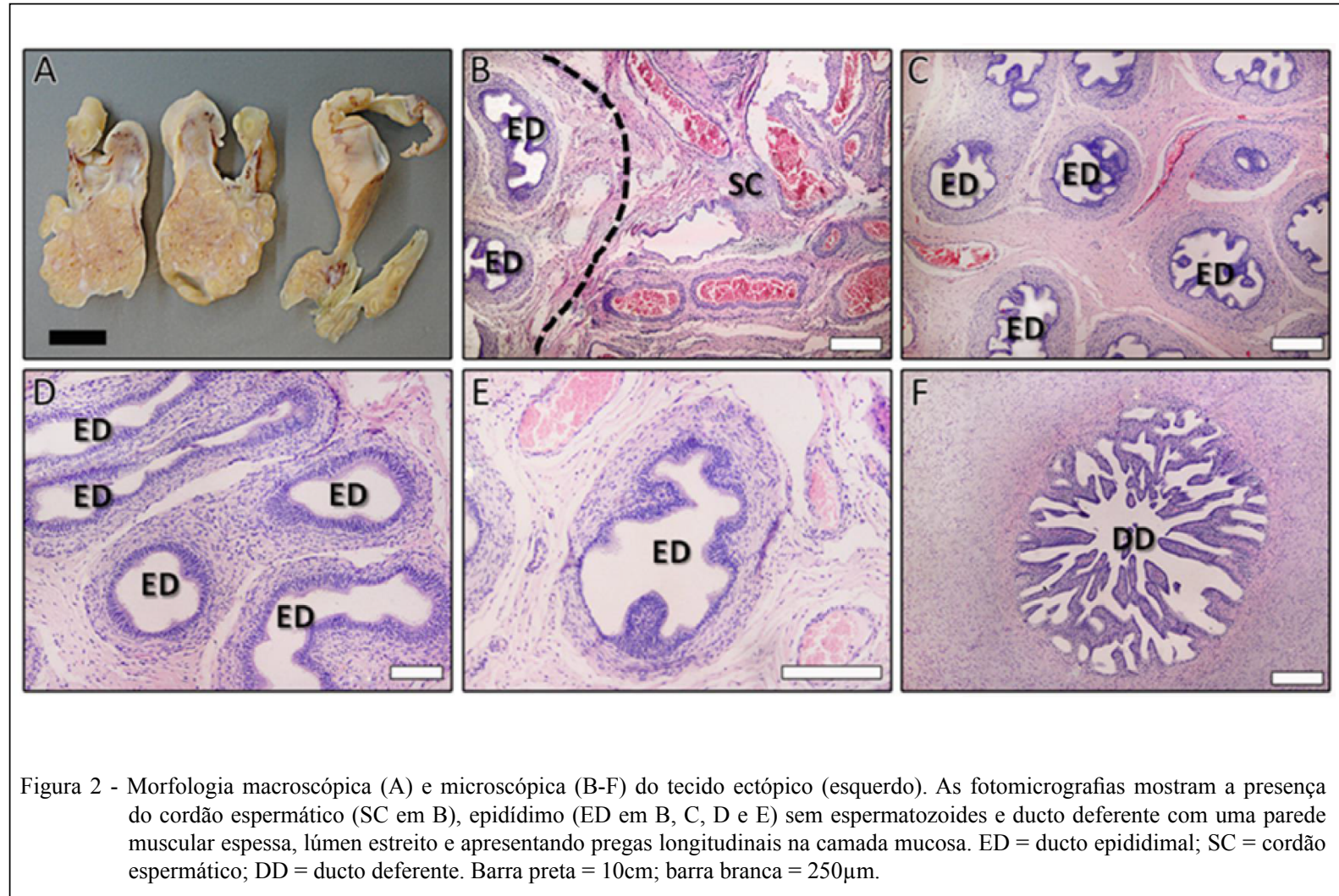

SCHUMACHER, 1992; PARKER et al., 1997; STRONG et al., 1997), e não foram encontrados relatos nos principais periódicos científicos nacionais. Nos trabalhos internacionais, o monorquidismo foi relatado nas seguintes raças: Puro Sangue Inglês, Appaloosa, Árabe e Pampa (GARLICK, 1952; SANTSCHI, et al., 1989; STRONG et al., 1997; PETRIZZI et al., 2004). No presente caso, trata-se de um equino oriundo do estado de Minas Gerais, sem raça definida, porém sem qualquer característica de raças internacionais já acometidas pela mesma afecção.

Uma vez que o criptorquidismo é relativamente comum em equinos nacionais, houve a preocupação no presente trabalho de se realizar o teste hormonal com estimulação de hCG para confirmar a ausência de tecido testicular. A concentração basal de testosterona pode variar muito nos equinos, levando a conclusões erradas a respeito da presença de tecido testicular. A concentração basal de testosterona associada à estimulação com hCG parece ser o teste mais utilizado e que possui maior confiabilidade nos resultados em equinos com mais de 18 meses de vida e, em países que têm um inverno muito frio, o teste possui maior confiabilidade nos meses mais quentes (ARIGHI et al., 1989, SCHUMACHER, 1992). Essa associação no teste hormonal também foi utilizada por STRONG et al. (1997) e PETRIZZI et al. (2004).

Vários acessos cirúrgicos podem ser utilizados para explorar a cavidade abdominal e diagnosticar criptorquidismo ou monorquidismo, como os acessos inguinal, parainguinal, flanco e paramediano (STRONG et al., 1997; PETRIZZI et al., 2004). No presente relato, foi utilizado o acesso inguinal sob anestesia geral, que permitiu tanto boa exploração da região inguinal externa, como fácil acesso para exploração caudal do abdômen. A descoberta da cauda do epidídimo envolta pela túnica vaginal junto ao anel inguinal externo, sem que houvesse qualquer tecido cicatricial, foi essencial para o diagnóstico transcirúrgico de monorquidismo e remoção do epidídimo hipoplásico. Este achado não seria possível se outra abordagem cirúrgica fosse escolhida.

De maneira semelhante ao presente trabalho, STRONG et al. (1997) diagnosticaram monorquidismo em dois equinos na Universidade 
de Sydney, após histórico, exame físico, exploração cirúrgica, teste hormonal, histórico após a castração e exame histopatológico em um dos casos. Porém, o acesso cirúrgico inicialmente utilizado foi o inguinal, mas, quando nenhum tecido testicular foi detectado, fizeram outro acesso, o parainguinal, para explorar o abdômen caudal e certificar a ausência do testículo. Já na Itália, PETRIZZI et al. (2004) utilizaram o acesso pelo flanco e fizeram laparoscopia para diagnosticar monorquidismo em um garanhão appaloosa, demonstrando que a laparoscopia pode auxiliar no diagnóstico de monorquidismo. Também foi necessário exame histopatológico para o diagnóstico definitivo.

Conclui-se que o monorquidismo, apesar de raro, também pode acometer equinos nacionais. A abordagem cirúrgica inguinal sob anestesia geral foi a melhor escolha, pois permitiu fácil acesso ao tecido do epidídimo hipoplásico, seguido de ampla exploração da parte caudal do abdome, achados que permitiram o diagnóstico transcirúrgico de monorquidismo. Os autores recomendam esta abordagem nos casos em que a confirmação da localização extra ou intraabdominal do testículo não puder ser confirmada via palpação ou outro exame complementar. Além disso, os exames histológicos e hormonais foram essenciais para o diagnóstico definitivo.

\section{AGRADECIMENTOS}

À Fundação de Amparo à Pesquisa do Estado de Minas Gerais (FAPEMIG), à Coordenação de Aperfeiçoamento de Pessoal de Nível Superior Setor (CAPES) e ao Conselho Nacional de Desenvolvimento Científico e Tecnológico $(\mathrm{CNPq})$ pelo apoio financeiro.

\section{REFEERÊNCIAS}

ADAMS, S.B. Cryptorchidectomy. In: WHITE N.A.; MOORE J.N. (Eds.). Current practice of equine surgery. Philadelphia: Lippincott, 1990. p. 722-726.
ARIGHI, M.; BOSU, W.T.K. Comparison of hormonal methods for diagnosis of cryptorchidism in horses. Journal of Equine Veterinary Science, v.9, p.20-26, 1989. Disponível em: <http://www.sciencedirect.com/science/ article/pii/S0737080689801121>. Acesso em: 15 dez. 2013. doi: 10.1016/S0737-0806(89)80112-1.

GARLICK, N.L. An unusual case of monorchidism in a stallion. Journal of the American Veterinary Medical Association, v.121, n.905, p.101-103, 1952.

PARKER, J.E.; RAKESTRAW,W.T.K. Intra-abdominal testicular torsion in a horse without signs of colic. Journal of the American Veterinary Medical Association, v.210, p.375-377, 1997. Disponível em: <http://www.ncbi.nlm.nih.gov/pubmed/9057921>. Acesso em: 15 dez. 2013. PMID: 9057921.

PARKS, A.H. et al. Monorchidism in the horse. Equine Veterinary Journal, v.21, n.3, p.215-217, 1989.

PETRIZZI, L. et al. Monorchidism in an appaloosa stallion. Veterinary Record, v.155, p.424-425, 2004. Disponível em: <http://veterinaryrecord.bmj.com/content/155/14/424. citation>. Acesso em: 27 nov. 2013. doi: 10.1136/ vr.155.14.424.

SANTSCHI, E.M. et al. Monorchidism in three colts. Journal of the American Veterinary Medical Association, v.194, n.2, p.265266, 1989.

SCHUMACHER, J. Surgical disorders of the testicle and associated structures. In: AUER, J.A. (Ed.) Equine surgery. Philadelphia: Saunders, 1992. p.674-703.

SEARLE, D. et al. Equine castration: review of anatomy, approaches, techniques and complications in normal, cryptorchid and monorchid horses. Australian Veterinary Journal, v.77, p.428-434, 1999. Disponível em: <http://onlinelibrary.wiley. com/doi/10.1111/j.17510813.1999.tb12083.x/stract?denied AccessCustomisedMessage $=\&$ userIsAuthenticated $=$ false.$>$. Acesso em: 27 nov. 2013. doi: 10.1111/j.1751-0813.1999. tb12083.x.

STRONG, M. et al. Monorchidism in two horses. Australian Veterinary Journal, v.75, p.33-35, 1997. Disponível em: $<$ http:// onlinelibrary.wiley.com/doi/10.1111/j.1751-0813.1997.tb15704.x/ abstract>. Acesso em: 27 nov. 2013. doi: 10.1111/j.17510813.1997.tb157 